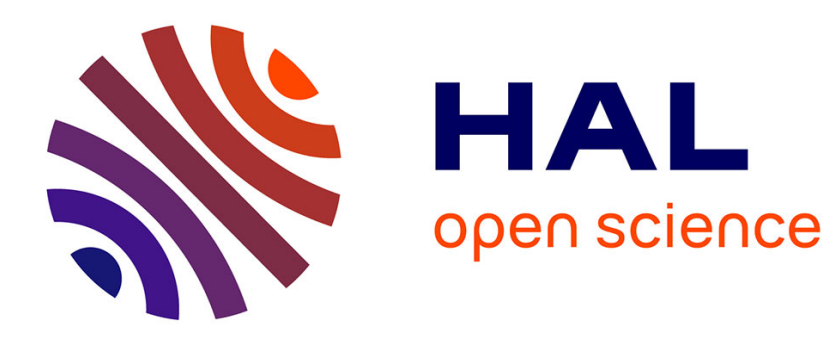

\title{
Phase transitions of binary mixtures in random media
}

\author{
F. Brochard, P.-G. de Gennes
}

\section{To cite this version:}

F. Brochard, P.-G. de Gennes. Phase transitions of binary mixtures in random media. Journal de Physique Lettres, 1983, 44 (18), pp.785-791. 10.1051/jphyslet:019830044018078500 . jpa-00232263

\section{HAL Id: jpa-00232263 https://hal.science/jpa-00232263}

Submitted on 1 Jan 1983

HAL is a multi-disciplinary open access archive for the deposit and dissemination of scientific research documents, whether they are published or not. The documents may come from teaching and research institutions in France or abroad, or from public or private research centers.
L'archive ouverte pluridisciplinaire HAL, est destinée au dépôt et à la diffusion de documents scientifiques de niveau recherche, publiés ou non, émanant des établissements d'enseignement et de recherche français ou étrangers, des laboratoires publics ou privés. 
Classification

Physics Abstracts

$64.70 \mathrm{~J}-68.10 \mathrm{C}$

\title{
Phase transitions of binary mixtures in random media
}

\author{
F. Brochard and P. G. de Gennes \\ Collège de France, 75231 Paris Cedex 05, France
}

(Reçu le 3 juin 1983, accepté le 20 juillet 1983)

Résumé. - Nous considérons un solide poreux, ou un gel, saturé par un système fluide à deux composants, $A, B$, dans le domaine de température où $A$ et $B$ démixent ; l'épaisseur $\xi$ de l'interface est supposée plus petite que la taille des pores. Le solide préfère être en contact avec la phase riche en $A$. Mais, si l'on fait décroître le potentiel chimique de B, la phase riche en $B$ pénètre la structure. Nous discutons la pénétration réversible, et trouvons deux modes distincts :

a) « invasion capillaire » (progressive).

b) " basculement " où tout le volume poreux est envahi soudainement (sauf peut-être pour une couche de mouillage d'épaisseur $\xi$ près du solide).

Les « champs » $H$ (reliés au potentiel chimique d'échange $\mathrm{A} / \mathrm{B}$ ) requis pour l'invasion capillaire $\left(H_{\mathrm{c}}\right)$ ou pour le basculement $\left(H_{\mathrm{f}}\right)$ sont dans le rapport

$$
H_{\mathrm{f}} / H_{\mathrm{c}} \cong(\xi / D)^{2-d_{\mathrm{f}}}
$$

où $d_{\mathrm{f}}$ est la dimension fractale de la surface. (Les gels de tiges rigides ont $d_{\mathrm{f}}=1$, alors que des gels flexibles en bon solvent ont $d_{\mathrm{f}}=5 / 3$.) Pour tous les gels le processus (b) doit dominer, et donne une transition du $1^{\mathrm{er}}$ ordre. Pour les solides poreux $\left(d_{\mathrm{f}}=2\right)$ une discussion plus détaillée est donnée sur un modèle de capillaires interconnectés (fins) à diamètre aléatoire : pour ce modèle, le processus (a) domine et il ne devrait pas y avoir de transition nette.

Abstract. - We consider a porous solid, or a gel, saturated by a two fluid system $A+B$, in the limit
where the thickness $\xi$ of the AB interface is smaller than the pore size $D$. The solid prefers to be in
contact with one of the fluids (A). But, if we decrease the chemical potential of B, the B fluid enters
the structure. We discuss the reversible penetration process, and find two distinct modes :

a) «Capillary invasion » with clusters of B regions growing progressively.

b) «Flip process » where all pores are abruptly invaded by B (except possibly for a thin sheath of thickness $\xi$ near the walls).

The « fields " $H$ ( = chemical potential changes $)$ required to perform capillary invasion $\left(H_{c}\right)$ or flip $\left(H_{\mathrm{f}}\right)$ are in ratio :

$$
H_{\mathrm{f}} / H_{\mathrm{c}} \cong(\xi / D)^{2-d_{\mathrm{f}}}
$$

where $d_{\mathrm{f}}$ is the fractal dimension of the pore surface. Gels made with rod-like molecules have $d_{\mathrm{f}}=1$, while flexible chains in good solvents have $d_{\mathrm{f}}=5 / 3$. For all gels the flip process should dominate, and we expect a sharp transition. On the other hand, porous solids $\left(d_{\mathrm{f}}=2\right)$ require a special discussion. Modeling the pores as interconnected capillaries of random diameter $D$, we find that capillary invasion dominates in this case, and we expect no sharp transition. 


\section{The relation with random fields.}

The behaviour of lutidine/water mixtures in agarose gels is currently being studied at Pittsburgh [1]. Similar experiments can (and should) be contemplated with a solid porous matrix.

The gel, or porous medium, has two main effects on the binary mixture A + B. (1) It prefers one component (say A) of the mixture ; (2) It depresses the transition temperature near the contact surface. Effect (1) is conceptually equivalent to a random field acting on a ferromagnet : the simplest case of random fields (where the scale of the randomness is smaller than the unperturbed correlation length) has been analysed theoretically [2-6] and some good experimental examples have been found [7]. The existence of a sharp transition (in 3d systems) is probable but is still a matter of debate. These discussions are relevant for our gels or porous systems, when the temperature $T$ is only slightly below the consolute point $T_{\text {co }}$ : the unperturbed correlation length $\xi(T)$ is then larger than the pore size $D$. Here we explore the opposite limit of lower temperatures $(\xi<D)$. Then we deal with thin interfaces, and can discuss their relation to the pores in terms of capillary equilibria.

The effect (2) is also important, and may change the nature of the wetting-non wetting transition at the walls [8-10]. Our discussion incorporates both (1) and (2). On the other hand, we assume that the pores are undeformable : with flexible polymer chains, the binary fluid reacts on the gel structure. Near $T_{\text {co }}$ this may lead to partial collapse of the gel [11-12]; but for $T<T_{\text {co }}$, these complications are expected to be minor.

\section{Porous media.}

The random pore model which we have in mind is classical. It is displayed in figure 1. Each link is a thin capillary of diameter $D$ length $L(\gg D)$, with a distribution of $D$ values. Different capillaries have uncorrelated $D$ values. Let us assume first that the capillary contact angle $\theta$ between $A$, B, and the solid wall, is finite (Fig. $2 a$ ). $\theta$ is smaller than $\pi / 2$ since the wall prefers A. Our aim is to decrease progressively the chemical potential of $B$, and to see if at some moment the fraction of B fluid increases abruptly.

This is reminiscent of the injection of a fluid B (such as mercury) in an empty non wetted porous system (e.g. : vycor glass). Macroscopic injection occurs abruptly as a percolation transition [13-

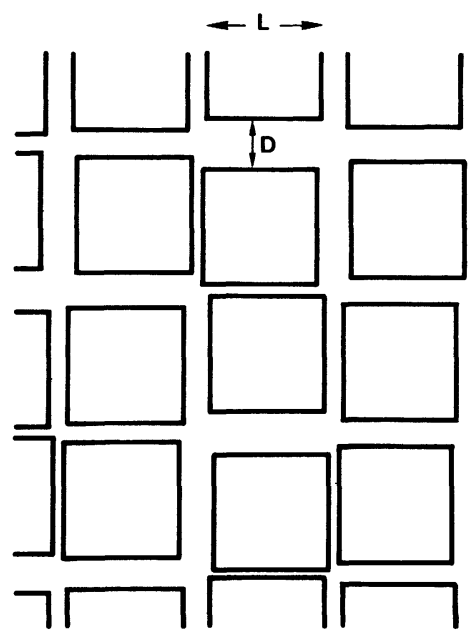

Fig. 1. - Capillary model for a porous solid : each capillary, or " pore " has a length $L$; but the diameters $D$ are randomly distributed. 


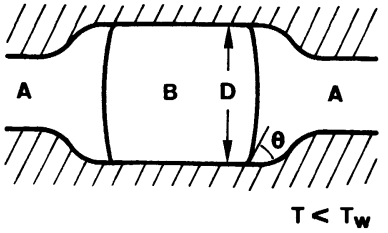

a)

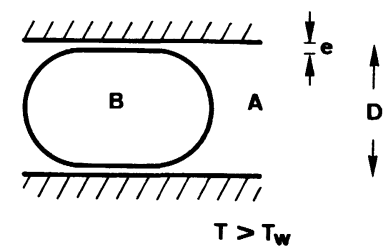

b)

Fig. 2. - A droplet appearing inside one pore : $a$ ) partial wetting ; b) complete wetting.

14]. The physics of the present problem is quite different. In injection experiments we are not dealing with thermodynamic equilibrium : a large pore can be reached by the mercury only if certain smaller pores which lead to it have also been invaded. In the present problem, a large pore can be filled with B (by diffusion of B molecules through A regions), even if all surrounding channels are too thin to accept a bulk B phase.

The Laplace equilibrium condition, for a cylindrical pore of diameter $D$, is :

$$
2 M_{0} H=\frac{2 \gamma}{R}=\frac{4 \gamma \cos \theta}{D}
$$

where we use the magnetic notation : $M_{0}(T)$ is the spontaneous magnetization at temperature $T$ and is related to the width of the $\mathrm{AB}$ coexistence curve. $H$ is an external field (related here to a chemical potential for $\mathrm{A} / \mathrm{B}$ exchange), $\gamma$ is the $\mathrm{AB}$ interfacial tension, and $R$ is the local curvature of the interface. For a given $H$, all pores of diameter $D>D(H)$ (where $D(H)$ is defined by Eq. 1) are filled. All pores $D<D(H)$ are empty.

Following these rules we generate a set of B clusters : they are connected pores, all filled with B, and they obey percolation statistics. Each cluster has a certain number of pores $(n)$ and is surrounded by a certain number of peripheral pores $(m)$. The corresponding statistical weight is $p^{n} q^{m}$ where $p$ and $q$ are functions of the field $H$

$$
p(H)=\int_{D(H)}^{\infty} w(D) \mathrm{d} H=1-q(H) .
$$

Here $w(D)$ is the distribution of pore sizes (each pore being weighted by it's own volume).

It is important to realize that this cluster structure is energetically stable. To understand this, consider first the simplest B cluster, i.e. a single pore with diameter $D>D(H)$. Filling up this pore with $B$ fluid, we gain an energy $E=E_{1}-2 E_{2}$

$$
\begin{aligned}
& E_{1}=\left(2 M_{0} H-\frac{4 \gamma \cos \theta}{D}\right) \frac{\pi}{4} L D^{2} \\
& E_{2}=\frac{\pi}{4} \cdot \frac{\gamma D^{2}}{\cos ^{2} \theta}(1-\sin \theta) .
\end{aligned}
$$

$E_{1}$ contains the effect of the pressure $M_{0} H$, and the effect of the solid-fluid interfaces $\gamma_{\mathrm{BS}}-\gamma_{\mathrm{AS}}=$ $\gamma \cos \theta$. The part $E_{2}$ is the energy of the fluid-fluid interface. For $D>D(H)$ the ratio $E_{1} / E_{2}$ is of order $L / D$ and is large $=$ the filled pore is stable. This argument can be extended to larger clusters, with arbitrary $n$ and $m$. The energy gain is then $n E_{1}-m E_{2}$. We now use the classical relation between averages $\langle n\rangle / p=\langle m\rangle / q$ and conclude that

$$
\langle n\rangle E_{1}-\langle m\rangle E_{2}=\langle n+m\rangle\left(p E_{1}-q E_{2}\right)
$$


thus (since $E_{1}>E_{2}$ ) for all finite $p_{1} q$, the energy gain is positive for the most relevant clusters, and they are stable.

Thus, when $H$ increases from 0 up, we generate B clusters. When $H$ reaches a certain value $H_{\mathrm{p}}$ such that $p\left(H_{\mathrm{p}}\right)=p_{\mathrm{c}}$ (the critical value for percolation) an infinite B cluster appears. But the field $H_{\mathrm{p}}$ is not associated with any thermodynamic singularity.

The average magnetization is :

$$
\frac{\bar{M}(H)}{M_{0}}=-1+2 \int_{D(H)}^{\infty} w(D) \mathrm{d} D .
$$

$\bar{M}(H)$ rises steeply when $D(H)$ is comparable to the average pore size, but keeps a finite slope in this region. Singularities may occur only at the ends $\left(D_{\min }, D_{\max }\right)$ of the distribution $w(D)$. The most important practical case would correspond to the existence of a $D_{\max }$. If $w\left(D>D_{\max }\right)=0$ and

$$
w\left(D \rightarrow D_{\max }\right) \simeq\left(D_{\max }-D\right)^{x}
$$

then, there is a special value $H_{\mathrm{m}}$ of the field, defined by $D\left(H_{\mathrm{m}}\right)=D_{\mathrm{max}}$, at which the B component first enters macroscopically. Near this point

$$
-\frac{\bar{M}}{M_{0}}+1=\text { (const.) }\left(H-H_{\mathrm{m}}\right)^{x} .
$$

Thus we expect at most a very weak singularity in $\bar{M}(H)$ (probably unobservable in practice) and this singularity should be entirely dependent upon the pore statistics.

These arguments have been formulated only for a highly idealized model of long cylindrical pores. But they are expected to be of more general validity. An essential point is that there is no coupling between adjacent B droplets : the distance between them is at least of order $L$ and the couplings (for the standard models of interfaces, ignoring long range Van der Waals forces) are exponentially small $(\sim \exp -L / \xi)$. Thus different droplets cannot switch on cooperatively.

Our discussion up to now assumed partial wetting (finite $\theta$ ). In some cases we may have complete wetting of the wall by A (Fig. 2b). The detailed shape of the B droplet near the walls is dependent on weak Van der Waals forces [15], but the curvature $R$ near the pore axis is still very near $D / 2$. Thus equation holds with $\theta=0$ and the discussion is not qualitatively modified, whenever the thickness of the wetting layers is much smaller than the pore size $D$.

\section{A gel of thin rods.}

Many gels are made of rigid chains, or bundles of chains. If the diameter $2 b$ of the bundles is much smaller than $\xi$, the arguments of section II break down.

1) Consider first a single bundle, and call $T_{\mathrm{A}}$ and $T_{\mathrm{B}}$ the free energy per unit length of bundle, when it is immersed in a bulk $\mathrm{A}$ or $\mathrm{B}$ phase. For $H=0$ a macroscopic interface cutting a simple bundle has the zero curvature shape of figure $3 a$, described by

$$
r(z)=\lambda \cosh \left(\frac{z}{\lambda}\right) \quad(r \gg \xi)
$$

where the balance of forces along the bundle axis imposes

$$
T_{\mathrm{B}}-T_{\mathrm{A}}=2 \pi \gamma \lambda \text {. }
$$

2) Consider now a droplet of $B$ in a random array of bundles (Fig. $3 b$ ). Near each bundle equation 5 prevails. Far from the bundle we have a weak curvature related to the field by Laplace's 


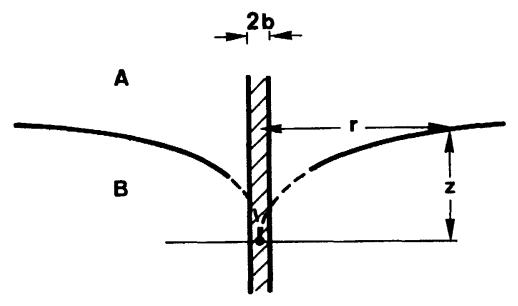

a)

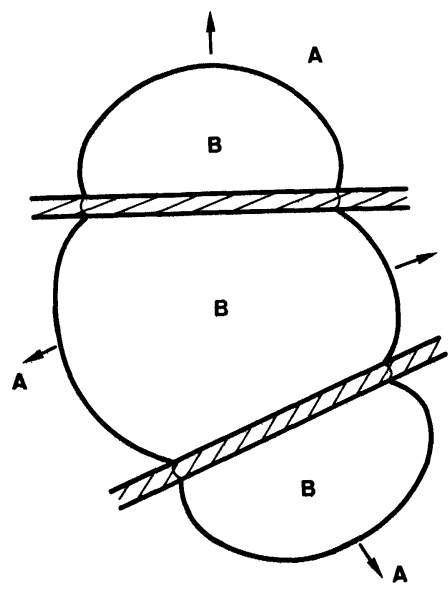

b)

Fig. 3. - Interactions between droplets and rods : $a$ ) a single rod with an $\mathrm{AB}$ interface which is (on the average) normal to the rod; $b$ ) a B droplet growing in an A medium : the droplet is not blocked by a random array of adverse rods.

equation. One essential difference with figure $1 a$ is that now the droplets originating from different "pores » are fused = thus the trend towards a cooperative transition is much stronger.

This also shows up if we think of a line dependent growth for a single droplet : starting from the centre of a " pore " we begin with a spherical droplet of radius $r<D$ but when we reach $r \sim D$, the droplet deforms and tends to surround the adjacent bundle, going through the conformation of figure $3 b$. The free energy in zero field $H$ is an increasing function of $r$ at $r \ll D$ and goes down precipitously for $r \sim D$ (coalesced droplets). Adding the field we are led to expect an abrupt transition, and following the argument of reference 2, we do not expect this to be significantly broadened by macroscopic inhomogeneities in the gel structure.

We discuss the energy balance in more detail in the next section.

\section{Two types of invasion.}

We now consider a more general porous structure, where the active surface $S$ has a certain fractal dimensionality $d_{\mathrm{f}}: d_{\mathrm{f}}=2$ for normal porous solids, $d_{\mathrm{f}}=1$ for rods, and $d_{\mathrm{f}}=5 / 3$ for polymer gels in good solvents $[16,17]$. Our earlier discussions led to two types of invasion by the B fluid under a «field " $H$. 
4.1 Capillary INVASION with droplets which fill only partly the pore space, as in figure 2. This is associated with fields of order :

$$
H_{\mathrm{c}}=\frac{\gamma}{D M_{0}(T)}
$$

where $D$ is now an average pore size.

4.2 FLIP PROCESS where essentially all of the pore space is suddenly invaded (except possibly for a thin melting sheath of order $\xi$ near the active surface). The corresponding field $H_{\mathrm{f}}$ is defined by

$$
2 M_{0} H_{\mathrm{f}} D^{3}=\tilde{\gamma} S
$$

where $\tilde{\gamma}=\gamma_{\mathrm{BS}}-\gamma_{\mathrm{AS}}$ is the energy per unit area required when the surface $S$ switches from an $\mathrm{A}$ environment to a B environment ( $\tilde{\gamma}$ depends on temperature, and on the strength of the surface fields). $S$ is the fractal area of one pore : it depends on the maximum scale $(D)$ in the structure, and on the minimum scale $\varepsilon$, according to [17]

$$
S=D^{d_{\mathbf{f}}} \varepsilon^{2-d_{\mathbf{f}}} .
$$

The detailed value of $\varepsilon$ depends on the strength of the random fields, but $\varepsilon \leqslant \xi$. (For qualitative purposes it is suggestive to take $\tilde{\gamma} \sim \gamma$ and $\varepsilon \sim \xi$ ).

Let us now compare the two fields

$$
\frac{H_{\mathrm{f}}}{H_{\mathrm{c}}} \cong\left(\frac{\varepsilon}{D}\right)^{2-d_{\mathrm{f}}} \frac{\tilde{\gamma}}{\gamma} .
$$

We see that, in the limit of interest (large $D), H_{\mathrm{f}}$ is smaller than $H_{\mathrm{c}}$ whenever the fractal dimension $d_{\mathrm{f}}$ is smaller than 2 : in this case the flip process will dominate, and will generate a sharp transition. On the other hand, for $d_{\mathrm{f}}=2$, equation 13 is ambiguous, but the discussion in section 2 suggests no transition for the specific model chosen $(L \gg D)$.

It may be worthwhile to note that, in a 2 dimensional world, equation 13 would be replaced by

$$
\frac{H_{\mathrm{f}}}{H_{\mathrm{c}}} \sim D^{d_{\mathrm{f}}-1} \quad(d=2)
$$

where $d_{\mathrm{f}}(\geqslant 1)$ is the fractal dimensionality of the pore perimeter. Thus, for $d=2$, whenever the pores are indeed fractal, we expect capillary invasion. The droplets trapped in different pores are uncoupled $=$ we expect no sharp transition for $d=2$.

Returning to 3 dimensions, we conclude that, inside a gel, a binary mixture should display a well defined coexistence curve. Of course, our discussion is restricted to thermodynamic equilibrium : it is clear that the capillary processes involved in the progression of an $A B$ interface may lead to strong irreversibility.

The present discussion was restricted to random systems. It is instructive to consider also the opposite limit of periodic structures (planes or rods) perturbing an AB phase transition. We analyse this case (for $D>\xi$ ) in a separate publication [18].

\section{Acknowledgments.}

We have greatly benefited from conversations and correspondence with W. Goldburg and J. Maher on gels; from discussions with E. Brezin, L. Pelliti, S. Leibler, B. Widom on wetting transitions, and with A. Aharony, M. Fisher, Y. Imry on random fields. 


\section{References}

[1] GoldBURG, W., MAHER, J., private communication.

[2] ImRY, Y., MA, S., Phys. Rev. Lett. 35 (1975) 1399.

[3] Aharony, A., Imry, Y., Ma, S., Phys. Rev. Lett. 37 (1976) 1364 ;

Young, A. P., J. Phys. C 10 (1977) L-257;

Parisi, G., Sourlas, N., Phys. Rev. Lett. 43 (1979) 744.

[4] Pytte, E., ImRy, Y., Mukamel, D., Phys. Rev. Lett. 46 (1981) 1173.

[5] Grinstein, G., Ma, S., Phys. Rev. Lett. 49 (1982) 685.

[6] Villain, J., J. Physique Lett. 43 (1982) L-551.

[7] Yoshizawa, H., Cowley, R., Shirane, G., Birgeneau, R., Guggenheim, H., Ikeda, H., Phys. Rev. Lett. 48 (1982) 438.

[8] Cahn, J. W., J. Chem. Phys. 66 (1977) 3667;

Pandit, R., Wortis, M., Phys. Rev. B 25 (1982) 3226.

[9] Moldover, M., CAHN, J., Science 207 (1980) 1073;

Kwon, O. et al., Phys. Rev. Lett. 48 (1982) 185;

Pohl, D., Goldburg, W., Phys. Rev. Lett. 48 (1982) 1111.

[10] Nakanishi, H., Fisher, M., Phys. Rev. Lett. 49 (1982) 1565 ;

BREZIN, E., HALPERIN, B., LeibleR, S., to be published.

[11] De Gennes, P. G., J. Physique Lett. 37 (1976) L-59.

[12] Brochard, F., DE GenNes, P. G., Ferroelectrics 30 (1980) 33.

[13] Chatzis, J., Dullien, F., J. Can. Pet. Technology 16 (1977) 97.

[14] De Gennes, P. G., Guyon, E., J. Mécanique 17 (1978) 403.

[15] De GenNes, P. G., J. Physique Lett. 42 (1981) L-377.

[16] De Gennes, P. G., Scaling Concepts in Polymer Physics (Cornell U.P., Ithaca) 1979.

[17] Mandelbrot, B., The fractal geometry of nature (Freeman, San Francisco) 1982.

[18] Brochard, F., De Gennes, P. G., submitted to C. R. Hebd. Séan. Acad. Sci. 